Cahiers
de la Recherche
sur les Droits

Cahiers de la recherche sur les droits

Fondamentaux fondamentaux

$14 \mid 2016$

Urbanisme et droits fondamentaux

\title{
Urbanisme et participation
}

Town-Planning and Public Consultation

Anne-Sophie Denolle et Eugénie Duval

\section{CpenEdition}

Journals

Édition électronique

URL : https://journals.openedition.org/crdf/585

DOI : $10.4000 /$ crdf.585

ISSN : 2264-1246

Éditeur

Presses universitaires de Caen

Édition imprimée

Date de publication : 1 novembre 2016

Pagination : 31-39

ISBN : 978-2-84133-838-2

ISSN : 1634-8842

Référence électronique

Anne-Sophie Denolle et Eugénie Duval, « Urbanisme et participation », Cahiers de la recherche sur les droits fondamentaux [En ligne], 14 | 2016, mis en ligne le 01 octobre 2019, consulté le 14 novembre 2022. URL : http://journals.openedition.org/crdf/585 ; DOI : https://doi.org/10.4000/crdf.585 


\title{
Urbanisme et participation
}

\author{
Anne-Sophie DENOLLE \\ Maître de conférences en droit public à l'université de Nîmes \\ Laboratoire détection, évaluation et gestion des risques CHROniques et éMErgents (CHROME, EA 7352) \\ Membre associé du Centre de recherche sur les droits fondamentaux et les évolutions du droit (CRDFED, EA 2132)
}

\section{Eugénie DUVAL}

Doctorante en droit public à l'université de Caen Normandie

Centre de recherche sur les droits fondamentaux et les évolutions du droit (CRDFED, EA 2132)

I. Un urbanisme appauvri en participation : la volonté du législateur?

A. État des lieux de la participation: un bilan mitigé

B. Une perception péjorative de la participation relayée par le législateur

\section{Une participation plurielle, dynamisée par ses acteurs au niveau local}

A. Une participation renforcée par l'action de certains élus

B. Les mécanismes parallèles de la participation:

le développement de formes d'actions citoyennes contestataires

L'urbanisme est sans aucun doute l'un des domaines de prédilection de la participation. À l'origine, les processus participatifs se déclinaient essentiellement via l'enquête publique. Or celle-ci révèle que la participation en droit de l'urbanisme était avant tout motivée par la protection du droit de propriété. Puis, progressivement sont apparues d'autres considérations qui ont transformé la finalité de la participation. D'abord, des préoccupations liées à l'environnement. Comme l'explique Jacques Caillosse en 1986,

À la relation qui prévalait jusqu'alors entre la procédure d'enquête et la défense de la propriété privée, la loi de juillet 1983 en substitue une autre: ce n'est plus l'atteinte éventuelle à la propriété privée qui conditionne le recours à la consultation publique, mais le sort réservé à l'environnement ${ }^{1}$.
Surtout, le développement des procédures participatives va être motivé par un autre impératif: celui de l'exigence démocratique qui passe par l'amélioration de la qualité de la décision publique. La participation a alors un objectif de taille, celui de pallier le désenchantement démocratique grandissant et, ainsi, de redonner un nouveau souffle à notre démocratie en modifiant la logique préexistante:

La promotion de l'idéologie de la participation a pour but de remédier à la perte de légitimité qui résulte de la crise de la représentation; il s'agit désormais de fonder les systèmes de pouvoir existants, non plus sur un principe transcendant, mais sur une pratique démocratique; la légitimité ne vient plus du sommet, de l'institution, mais de la base, des membres².

1. J. Caillosse, «Enquête publique et protection de l'environnement», Revue juridique de l'environnement, vol. 11, $\mathrm{n}^{\circ}$ 2, $1986, \mathrm{p} .152$.

2. J. Chevallier, «Réflexions sur l'idéologie de l'intérêt général», in Variations autour de l'idéologie de l'intérêt général, Centre universitaire de recherches sur l'action publique et le politique, épistémologie et sciences sociales (dir.), Paris, PUF, vol. I, 1978, p. 44 
Cette évolution se traduit, à partir des années 19801990, par une multiplication des instruments visant à promouvoir la participation, censée permettre au public d'être associé de manière effective à l'élaboration des décisions publiques ${ }^{3}$. À la traditionnelle enquête publique, sont venues s'ajouter la procédure de concertation ou encore celle du débat public, créant ainsi une véritable «offre» participative. Ces procédures réglementées principalement par le législateur ont eu un retentissement particulier dans le domaine de l'urbanisme, celles-ci ayant vocation à s'appliquer aux projets d'aménagement et de construction. Le droit de l'urbanisme apparaît alors comme un terreau fertile au développement de l'idée participative en France: en raison de son ancienneté puisque l'urbanisme éprouve depuis plus de trente ans la participation, mais également en raison de l'importance de son champ d'application, de nombreux projets d'aménagement étant concernés.

Ainsi, tout laisse à penser que le droit de l'urbanisme est un modèle à suivre en la matière, qu'il pourrait être transposé aux autres domaines de l'action publique. L'étude des formes réglementées de participation montre que celleci est pourtant loin d'être optimale (I). Si la participation réglementée s'avère relativement décevante, certaines autorités publiques et certains citoyens semblent se réapproprier cette idée de participation et la font vivre, montrant ainsi que la participation est une notion plurielle (II).

\section{Un urbanisme appauvri en participation: la volonté du législateur?}

Les procédures participatives applicables en matière d'urbanisme sont aujourd'hui nombreuses, de telle sorte que la plupart des décisions prises dans ce domaine qui présente un certain enjeu pour les riverains et leur cadre de vie est couverte par ces procédures. Toutefois leur efficacité est relative $(\mathrm{A})$. Le législateur s'est récemment emparé de ce constat - dont il semble d'ailleurs oublier qu'il en est en partie responsable - pour relayer une image plutôt péjorative de la participation et ainsi justifier sa rationalisation (B).

\section{A. État des lieux de la participation: un bilan mitigé}

Il existe aujourd'hui une diversité de procédures participatives applicables en matière d'urbanisme. Mais chacune souffre de lacunes que le législateur n'a jamais vraiment cherché à combler, lacunes qui, pour certaines procédures, sont pourtant dénoncées depuis longtemps par la doctrine ${ }^{4}$.

Ainsi, l'enquête publique, procédure ancienne et multiforme en droit de l'urbanisme ${ }^{5}$, n'est toujours pas en mesure de garantir une réelle prise en compte de l'avis du public dans l'élaboration des décisions administratives. L'enquête publique intervient en effet très tardivement dans le processus d'élaboration de la décision, à un stade où il n'est plus permis de discuter les différentes options, où le projet de construction ou d'aménagement soumis à autorisation est présenté sous sa forme quasi définitive ne laissant que peu de place à son éventuelle évolution.

L'enquête publique a pour particularité d'être diligentée par un commissaire-enquêteur chargé de veiller à son bon déroulement, de recueillir l'opinion du public, d'émettre un avis sur le projet. Mais l'avis du commissaire-enquêteur n'est pas tenu de refléter celui du public et l'autorité décisionnelle n'est aucunement tenue de le suivre, ni même de se prononcer sur cet avis ${ }^{6}$.

En 2008, Louis-Narito Harada avait évalué le niveau participatif de la procédure d'enquête publique environnementale à l'aide d'outils sociologiques de référence développés par l'américaine Sherry Arnstein?. Il en avait conclu que l'enquête publique relevait de la « coopération symbolique $»^{8}$.

Huit ans plus tard, tout laisse à penser que c'est le même constat qui pourrait s'opérer, la procédure d'enquête publique n'ayant que peu évolué.

3. Nous utiliserons ici essentiellement l'expression "participation du "public" " puisque le droit de l'urbanisme mobilise un champ assez large de participants, ne se cantonnant pas aux seules personnes ayant la qualité de citoyens au sens juridique du terme, c'est-à-dire au sens d'électeurs.

4. Voir notamment P. Idoux, «Les eaux troubles de la participation du public», Environnement, juillet 2005, p. 89; E. Le Cornec, «Construire des quartiers durables: du concept au projet urbain. La participation du public», Revue française de droit administratif, 2006, p. 770-774; B. Delaunay, "Le débat public», L'actualité juridique. Droit administratif, n 42, décembre 2006, p. 2322-2327; J.-C. Hélin, "La participation du public aux décisions en matière d'urbanisme: une intégration ambiguë?», Construction - Urbanisme, juillet 2007, p. 15-21.

5. Il existe aujourd'hui trois types d'enquêtes publiques, celles soumises au Code de l'environnement (les enquêtes publiques environnementales aussi dénommées «enquêtes de la loi Bouchardeau»), les enquêtes relevant du Code de l'expropriation (art. L. 110-1) qui se déroulent préalablement aux déclarations d'utilité publique sauf lorsque le projet est susceptible de porter atteinte à l'environnement et enfin les enquêtes régies par le Code des relations entre le public et l'Administration (art. L. 134-1). Pour en savoir plus sur la cohabitation de ces trois régimes, voir P. Bon, "L'association du public aux décisions prises par l'administration», Revue française de droit administratif, 2016, p. 27-35. L'auteur précise que «le déroulement des enquêtes du code des relations entre le public et l'administration, tout comme celui des enquêtes du code de l'expropriation, offre au public un peu moins de garanties que celui des enquêtes environnementales (mais, en sens inverse, les enquêtes environnementales sont sensiblement plus lourdes à organiser)» (p. 34).

6. CE, 15 décembre 2015, Commune de Saint-Cergues, $\mathrm{n}^{\circ} 374027$

7. L.-N. Harada, «Démocratie et environnement: l'exemple de l'enquête publique en France», Droit de l'environnement, $\mathrm{n}^{\circ}$ 156, mars 2008 , p. 7-9. À la fin des années 1960, Sherry Arnstein a élaboré une échelle de la participation: de la "manipulation», considérée comme de la "nonparticipation", à l'idée en haut de l'échelle d'un "citizen control» ou d'un "delegated power», on retrouve des niveaux intermédiaires parmi lesquels la consultation, qualifiée de participation symbolique («tokenism») (S. R. Arnstein, «A Ladder of Citizen Participation», Journal of the American Planning Association, vol. $35, \mathrm{n}^{\circ}$ 4, juillet 1969, p. 217).

8. Ibid., p. 9 . 
Le législateur a tout de même tenté de remédier à certaines des lacunes de la procédure, sans la réformer directement, mais en créant d'autres procédures susceptibles d'intervenir en amont de l'enquête publique.

La phase de concertation du public est ainsi devenue obligatoire en droit de l'urbanisme'. Mais si cette procédure a le mérite d'intervenir à un stade plus précoce de l'élaboration des projets de construction et d'aménagement, elle est assortie de garanties nettement insuffisantes pour permettre une participation effective du public ${ }^{10}$. En effet, les modalités d'organisation de la concertation sont laissées à la discrétion de l'autorité publique. La concertation peut se traduire par un simple affichage, une exposition, une maquette, une réunion. Si le public peut en principe formuler des remarques et propositions, aucun instrument précis pour recueillir et consigner cet avis n'est envisagé ${ }^{11}$. La concertation prend ainsi, le plus souvent, la forme d'une information pédagogique ${ }^{12}$.

Une autre procédure peut intervenir en amont de l'enquête publique mais uniquement pour les projets d'aménagement ou d'équipement d'intérêt national. Il s'agit du débat public. Bien plus élaboré que le mécanisme de la concertation, le débat public est accompagné d'un certain nombre de garanties. Il est notamment organisé sous le contrôle d'une autorité administrative indépendante: la Commission nationale du débat public (CNDP). Mais la tenue du débat public demeure facultative. La CNDP dispose en effet d'un pouvoir discrétionnaire pour décider de l'opportunité d'organiser le débat. S’il a lieu, la CNDP en rédigera un bilan transmis au maître d'ouvrage et censé, si nécessaire, l'aider à reconsidérer son projet. Le porteur de projet devra sur le fondement de ce bilan prendre une décision motivée indiquant s'il souhaite poursuivre le projet et, le cas échéant, les conditions de sa mise en œuvre. Mais le maître d'ouvrage n'est pas contraint d'apporter des réponses concrètes aux difficultés soulevées, même celles qui apparaissent de manière récurrente. Et malgré des opinions majoritairement opposées au projet, le maître d'ouvrage n'est pas tenu de le modifier pour prendre en compte l'avis du public.
En somme, pour reprendre les termes de Bénédicte Delaunay, le débat public

[...] ne garantit que le droit d'être informé et de participer à la discussion qui précède la décision. [Il] ne garantit nullement une prise en compte des points de vue exprimés et ne permet pas nécessairement d'associer le citoyen à la décision collective ${ }^{13}$.

Concernant les projets de construction, de travaux, d'ouvrage ou d'aménagement qui n'entrent pas dans le champ d'application du débat public, de la concertation ou de l'enquête publique, la loi Grenelle II a instauré le système de mise à disposition du public dès lors que le projet est soumis à étude d'impact ou évaluation environnementale ${ }^{14}$. Il s'agit, dans un délai relativement court de quinze jours minimum, de permettre au public avant la décision finale de prendre connaissance du projet. Les propositions et observations du public doivent pouvoir s'exprimer et peuvent être consignées dans un registre. La procédure peut être assimilée à une enquête publique simplifiée qui se déroule dans des délais deux fois plus courts, sans commissaire-enquêteur et selon des modalités librement choisies par l'autorité publique ${ }^{15}$. Difficile là encore d'envisager que le public dispose des meilleures conditions pour se forger une opinion, l'exprimer et espérer la voir prise en compte.

Un espoir d'amélioration des procédures participatives a pu naître avec la loi du 27 décembre 2012 tendant à mettre en œuvre le principe de participation du public consacré à l'article 7 de la Charte de l'environnement ${ }^{16}$. Cette loi prévoit notamment que l'avis qui est exprimé par le public doit faire l'objet d'une synthèse qui dans certains cas devra être réalisée par une personnalité qualifiée. Il est également prévu la communication des motifs de la décision, la publication de la synthèse des avis exprimés dans laquelle doivent figurer ceux dont l'autorité décisionnelle a tenu compte $^{17}$. Cette synthèse a un intérêt majeur puisqu'elle permet de mesurer les effets d'une procédure participative par laquelle le public peut alors espérer influencer la décision finale ${ }^{18}$.

9. Loi n $85-729,18$ juillet 1985 relative à la définition de principes d'aménagement, JORF, 19 juillet 1985, p. 8152. La procédure est régie par l'article L. 103-2 du Code de l'urbanisme.

10. Pour une analyse critique récente de la procédure de concertation du public, voir B. Delaunay, «Les principes en droit de l'urbanisme. Le principe de participation du public», Construction - Urbanisme, novembre 2015, p. 9-14.

11. L'article L. 103-4 du Code de l'urbanisme dispose que «les modalités de la concertation permettent, pendant une durée suffisante et selon des moyens adaptés au regard de l'importance et des caractéristiques du projet, au public d'accéder aux informations relatives au projet et aux avis requis par les dispositions législatives ou réglementaires applicables et de formuler des observations et propositions qui sont enregistrées et conservées par l'autorité compétente».

12. Voir infra.

13. B. Delaunay, «Le débat public», p. 2327.

14. Loi $\mathrm{n}^{\circ}$ 2010-788 du 12 juillet 2010 portant engagement national pour l'environnement. Art. L. 122-1-1 et L. 122-8 du Code de l'environnement.

15. Pour une critique de cette procédure, voir R. Hostiou, «La loi du 12 juillet 2010 portant engagement national pour l'environnement: les modifications apportées au droit des enquêtes publiques ", Droit administratif, décembre 2010, p. 19-23.

16. Loi n ${ }^{\circ}$ 2012-1460 du 27 décembre 2012 relative à la mise en œuvre du principe de participation du public défini à l'article 7 de la Charte de l'environnement, JORF, $\mathrm{n}^{\mathrm{o}}$ 302, 28 décembre 2012, p. 20578. Cette loi a été complétée par l'ordonnance nº $2013-714$ du 5 août 2013 relative à la mise en œuvre du principe de participation du public défini à l'article 7 de la Charte de l'environnement, JORF, n 181,6 août 2013, p. 13396.

17. Art. L. 120-1, II, al. 6 et 8 du Code de l'environnement.

18. Voir notamment M.-C. Runavot, «La loi du 27 décembre 2012 relative à la mise en œuvre du principe de participation du public défini à l’article 7 de la Charte de l'environnement ou les variations autour du thème de la démocratie», Droit administratif, mai 2013, p. 16: «Le "tenu compte" semble impliquer, au-delà de la prise en compte et a fortiori de la prise en considération, l'obligation positive de l'Administration d'intégrer les observations dans le dispositif de la loi, d'infléchir voire de modifier le projet de texte dans l'un au moins des sens suggérés par le public. Avec 
L'apport de la loi de 2012 mérite toutefois d'être très largement nuancé, en particulier en matière d'urbanisme, puisque le nouveau mécanisme participatif envisagé ne vient pas compléter les procédures existantes. Il n'a en effet vocation à s'appliquer que pour les décisions ayant une incidence sur l'environnement qui ne sont pas déjà régies par l'une des procédures prévues par la loi. Le nouveau mécanisme ne pourra donc concerner que de manière extrêmement marginale les mesures d'urbanisme affectant l'environnement qui sont largement couvertes par les processus participatifs préexistants.

Le bilan des procédures participatives est assez décevant dans la mesure où elles ne parviennent pas à offrir les garanties pour le public d'une réelle prise en considération de son opinion, principe qui relève pourtant du bon sens, et, en droit de l'environnement au moins, de plusieurs textes, notamment la convention d'Aarhus de $1998^{19}$.

Le non-respect de cette exigence tend dans tous les cas à dévaloriser les processus participatifs aux yeux du public et des autorités amenées à les utiliser. Le législateur, au lieu de remédier à cette situation, semble aujourd'hui s'en prévaloir pour motiver une simplification, un amoindrissement des exigences participatives dont l'utilité est ainsi questionnée.

\section{B. Une perception péjorative de la participation relayée par le législateur}

Pour l'année 2015, le législateur ou son mandaté - le gouvernement, par la voie de l'ordonnance - va intervenir à deux reprises dans le champ des procédures participatives mais dans deux registres bien différents.

Il va engager une réforme lançant la création du Code des relations entre le public et l'Administration qui aurait pu être l'occasion d'améliorer les mécanismes de la participation. Le législateur avait ainsi habilité le gouvernement à procéder par ordonnance à l'adoption de la partie législative du nouveau Code et l'autorisait pour ce faire à « apporter aux règles de procédure administrative non contentieuse les modifications nécessaires pour renforcer la participation du public à l'élaboration des actes administratifs ${ }^{20}$.

Mais l'ordonnance prise le 23 octobre 2015 va se contenter d'effectuer une codification globalement à droit constant, loin de l'ambition affichée par la réforme législative initiale ${ }^{21}$.

C'est à travers une tout autre initiative, inspirée d'exigences bien éloignées de celles liées à l'impératif démocratique, que le gouvernement va suggérer au législateur la modification des dispositions relatives à la participation du public. C'est en effet par le biais de son projet de loi relatif à la croissance, l'activité et l'égalité des chances économiques que le gouvernement va proposer au législateur de faire évoluer les processus participatifs. Le législateur validera le principe et décidera de confier au gouvernement le soin de préciser par ordonnances les modalités de la participation rénovée ${ }^{22}$.

Il va tout de même poser un cadre général qui, du fait de l'objet particulier de la loi, oblige à appréhender la participation sous un angle inhabituel qui n'est pas celui de la protection de l'environnement, ni celui de l'exigence démocratique mais celui de la performance économique.

Dans ce schéma de pensée, la participation est nécessairement perçue de manière péjorative, comme chronophage, coûteuse et ralentissant les ambitions du développement économique. Cette vision est relayée par la loi Macron dont l'objet comme nous le rappelle Pascal Planchet est «clairement de revoir les règles de droit dans ces deux matières [l'environnement et l'urbanisme] soupçonnées de faire obstacle à l'activité économique $»^{23}$.

cette procédure, l'autorité de décision ne pourrait donc pas se contenter de consulter les administrés et demeurer libre d'ignorer les opinions émises au moment de décider. En ce sens, la nouvelle procédure deviendrait participative en ce qu'elle assurerait une emprise des administrés sur la décision et constituerait une plus-value démocratique par rapport à l'Administration consultative». L'auteur invite toutefois à la prudence et retient qu' «il reviendra à la pratique, et en particulier au juge, de donner à l'expression une signification plus ou moins contraignante pour l'Administration. À l'évidence, la chose ne sera pas aisée. [...] comment le juge pourra-t-il matériellement toutes les consulter [les observations du public] et les confronter à la teneur de la décision prise sans dépasser des délais raisonnables de jugement? Dans quelle mesure également l'Administration devra-t-elle intégrer ces observations? Tenir compte de l'une d'elles seulement sur les quelques centaines formulées sera-t-il suffisant? L'Administration devra-t-elle, inversement, justifier sa position à l'égard de chacune des observations, auquel cas la loi de 2012 fonderait une nouvelle obligation de motivation?».

19. Convention d'Aarhus sur l'accès à l'information, la participation du public au processus décisionnel et l'accès à la justice en matière d'environnement du 25 juin 1998, ratifiée par la France au titre de la loi nº 2002-285 du 28 février 2002. Voir plus particulièrement l’article 6, $\$ 8$ de la convention : «Chaque Partie veille à ce que, au moment de prendre la décision, les résultats de la procédure de participation du public soient dûment pris en considération » et l'article $6, \S_{4}$ : «Chaque Partie prend des dispositions pour que la participation du public commence au début de la procédure, c'est-à-dire lorsque toutes les options et solutions sont encore possibles et que le public peut exercer une réelle influence».

20. Loi n⿳⺈ 2013-1005 du 12 novembre 2013 habilitant le gouvernement à simplifier les relations entre l'Administration et les citoyens, art. 3 , III. Pour un commentaire de la loi, voir P. Bon, «L'association du public...»; S. Saunier, «L'association du public aux décisions prises par l'administration », L'actualité juridique. Droit administratif, nº 43, décembre 2015, p. 2426-2432.

21. Ordonnance $\mathrm{n}^{\circ}$ 2015-1341 du 23 octobre 2015 relative aux dispositions législatives du Code des relations entre le public et l'Administration, JORF, $\mathrm{n}^{\circ} 248,25$ octobre 2015, p. 19872. Sébastien Saunier résume ainsi la portée de la réforme: «En somme, le code opère largement une réforme en trompe-l'œil: les occasions manquées sont multiples, tandis que les avancées restent très partielles» (S. Saunier, «L'association du public... », p. 2432).

22. Loi $\mathrm{n}^{\circ}$ 2015-990 du 6 août 2015 pour la croissance, l'activité et l'égalité des chances économiques, JORF, n 181,7 août $2015, \mathrm{p}$. 13537, dite loi Macron. Pour un commentaire de la loi et ses implications en droit de l'urbanisme, voir P. Billet, «Le droit de l'urbanisme dans la loi Macron», La semaine juridique, édition administrations et collectivités territoriales, no 8, 29 février 2016, p. 36-44.

23. P. Planchet, «Quand les droits de l'urbanisme et de l'environnement font cause commune», L'actualité juridique. Droit administratif, $\mathrm{n}^{\circ} 39$, novembre 2015, p. 2194. 
La loi Macron propose ainsi de rationaliser les procédures participatives et non à proprement dit de les améliorer au profit de la protection de l'environnement et de l'expression démocratique.

Le législateur émet une série de consignes adressées au gouvernement pour «moderniser» et «simplifier» les procédures destinées à assurer l'information et la participation du public. Il invite d'abord à procéder à une harmonisation et une simplification de certaines procédures existantes, notamment celles instaurées par la loi du 27 décembre $2012^{24}$.

Il prévoit également la nécessité de simplifier et clarifier les enquêtes publiques et de développer le principe d'une procédure unique de participation pour plusieurs projets, plans ou programmes dont on suppose qu'ils seraient liés entre eux. Il s'agirait à travers cette dernière proposition de gagner du temps en effectuant une seule consultation du public sur un ensemble de projets, de décisions, ce qui aurait pour effet d'occulter la spécificité de chacun des projets, sans pour autant assurer une participation plus effective et plus rapide, la densité des documents à consulter pour le public rendant difficile une franche réduction des délais ${ }^{25}$.

La loi Macron invite en outre à admettre la possibilité d'adapter les procédures participatives à certains plans, programmes, projets afin de prendre en compte leurs caractéristiques, ce qui revient ici, comme le souligne Pascal Planchet, à ouvrir «la voie à un allégement des formalités de consultation du public $»^{26}$.

Enfin, le législateur promeut l'émergence de nouvelles procédures participatives,

[...] notamment des concertations préalables aux procédures de participation existantes, susceptibles d'être mises en œuvre par un droit d'initiative pouvant être ouvert notamment au public, à des associations et fédérations de protection de l'environnement, à des collectivités territoriales, à l'autorité compétente pour prendre la décision et au maître d'ouvrage, ainsi qu'une procédure de consultation locale des électeurs d'une aire territoriale déterminée sur les décisions qu'une autorité de l'État envisage de prendre sur une demande relevant de sa compétence et tendant à l'autorisation d'un projet susceptible d'avoir une incidence sur l'environnement ${ }^{27}$.
La procédure de consultation locale fait notamment écho à la situation du projet d'aéroport de Notre-Damedes-Landes pour lequel un référendum local devrait être organisé. Il s'agit ici de contourner la prétendue contradiction entre l'intérêt national du projet et l'ancrage local du référendum qui dans tous les cas ne sera qu'un référendum consultatif et non décisionnel.

De manière générale, la participation apparaît dans la loi Macron comme devant être rationnalisée, assouplie, suggérant qu'elle constitue, sous ses formes actuelles, une contrainte susceptible de limiter les projets de développement économique et de construction ${ }^{28}$.

Il s'agit toutefois d'une vision à court terme, car l'idéal démocratique n'est pas forcément contraire au développement économique, ni étranger à toute notion d'efficacité.

L'un des effets escomptés de la participation est d'ailleurs de permettre une meilleure application des dispositions objets de la participation. Tout laisse en effet à penser que le public qui aura participé à l'élaboration d'une décision publique en ayant eu la possibilité de donner son point de vue et de le voir pris en compte, sera, à n'en pas douter, plus enclin à respecter la décision publique.

Pour atteindre ces bienfaits, encore faut-il bien sûr que la participation soit optimale et permette une réelle prise en compte de l'avis du public. Mais comme on a eu l'occasion de le souligner supra, le législateur a préféré une participation plus souple moins contraignante pour l'autorité décisionnelle et peu efficace aux yeux du public. Ainsi dévoyée, la participation accompagnée de son lot de «lourdeur procédurale» ne peut que céder face aux impératifs d'efficacité économique qui lui sont présentés comme contradictoires. Le législateur saisit ici l'occasion de réformer les mécanismes participatifs pour les rationnaliser, les simplifier, en espérant que le gouvernement ne les vide pas totalement de leur substance. La situation est en tout cas assez révélatrice de la schizophrénie ambiante du législateur, qui prône le démantèlement d'un modèle participatif dont il a lui-même provoqué l'inefficience.

S'instaure de ce fait une certaine défiance à l'égard du législateur dans sa capacité à faire progresser la participation. Certaines autorités publiques, certains administrés ou groupes d'administrés ont d'ailleurs fait le choix de

24. Loi n 2012-1460 du 27 décembre 2012 relative à la mise en œuvre du principe de participation du public défini à l'article 7 de la Charte de l'environnement, évoquée supra.

25. Philippe Billet considère que l'incitation à mener des enquêtes conjointes, «avec des milliers de pages à lire dans un temps limité, conduit à s'interroger sur la pertinence de l'exercice et la réalité matérielle de la participation du public» (P. Billet, «La simplification du droit dans toute sa complexité. À propos des projets de simplification en matière d'urbanisme, d'environnement et de patrimoine culturel », La semaine juridique, édition administrations et collectivités territoriales, $n^{\circ} 8,23$ février 2015, p. 47). La loi Macron préconise en outre le recours systématique aux nouvelles technologies de l'information et de la communication. Mais comme le souligne Philippe Billet, «l'expérience de la dématérialisation met en évidence la fracture numérique et, en réalité, un échange des plus limités et une réduction du rôle de médiateur du commissaire enquêteur » (ibid., p. 49).

26. P. Planchet, "Quand les droits de l'urbanisme...", p. 2196.

27. Loi no 2015-990 du 6 août 2015 pour la croissance, l'activité et l'égalité des chances économiques, art. 106, I, $3^{\circ}, \mathrm{C}$.

28. Pascal Planchet conclut son analyse sur la loi Macron en ces termes: «En dépit de l'étendue de l'habilitation, force est de constater que le grand soir de la participation n'est pas venu. [...] Face à une telle profusion, il est difficile de tirer une conclusion d'ensemble si ce n'est que la loi Macron restera le symbole d'une approche économique décomplexée de l'urbanisme et de l'environnement» (P. Planchet, "Quand les droits de l'urbanisme... », p. 2199). 
s'approprier l'outil participatif pour le faire vivre, sans attendre l'injonction du législateur.

\section{Une participation plurielle, dynamisée par ses acteurs au niveau local}

Au niveau local, élus et citoyens sont en mesure de donner un nouveau souffle à l'idée de participation, de la dynamiser, révélant ainsi son caractère pluriel. D’abord, les textes laissant souvent une marge de manœuvre importante, les élus développent parfois des démarches plutôt ambitieuses afin de concrétiser cette exigence participative (A). Ensuite, il arrive que les citoyens s'invitent eux-mêmes dans les processus de décision, recourant ainsi à des modes de participation contestataires, plus informels (B).

\section{A. Une participation renforcée par l'action de certains élus}

Alors qu'il existe un certain nombre de textes prévoyant l'association des habitants à l'élaboration ou à la prise d'une décision, l'organisation même de cette participation est souvent laissée à la discrétion des autorités administratives. Or, comme nous avons pu le constater, celles-ci ne l'envisagent pas toutes de la même manière. Dans la concrétisation pratique de l'idée participative, certaines se contentent du strict minimum. D'autres au contraire ont une vision plutôt ambitieuse et n'hésitent pas à mettre en place des procédés originaux et ainsi, à renforcer ou rénover la participation.

L'élaboration des plans locaux d'urbanisme (PLU) est obligatoirement soumise à une procédure de concertation $^{29}$. Toutefois, le Code de l'urbanisme laisse une marge de manœuvre non négligeable à l'organe délibérant de la collectivité dans la détermination de son contenu. En effet,

À la différence du droit des enquêtes publiques pour lesquelles les modalités de la participation du public sont étroitement définies et réglementées [...], celles de la concertation ont fait l'objet d'une définition textuelle $a$ minima, l'idée étant de laisser aux autorités locales une large liberté d'appréciation pour définir ces règles, en fonction des circonstances et de l'importance du projet ${ }^{30}$.
L'article L. 103-4 dispose simplement que le public doit pouvoir "accéder aux informations relatives au projet et aux avis requis par les dispositions législatives ou réglementaires applicables et [...] formuler des observations et propositions ${ }^{{ }^{31}}$. Les pratiques sont alors variables, certains se bornent à organiser quelques réunions publiques, dans lesquelles la parole est plus ou moins libre, mais d'autres semblent avoir à cœur de rendre la participation plus effective. Par exemple, dans le cadre de l'élaboration de son PLU, la commune de Morsang-sur-Orge en région parisienne se targue d'avoir mis en place un «PLU $100 \%$ participatif», associant le public dans le cadre d'un «Conseil local d'élaboration du PLU ${ }^{32}$ composé de quatre-vingts personnes. Elle inscrit ainsi la participation dans un temps plus long, ponctué de restitutions publiques auprès des habitants. Il s'agit d'une démarche ambitieuse ${ }^{33}$ montrant que les collectivités souhaitent parfois imaginer des dispositifs nouveaux. On pourrait toutefois penser que certaines démarches, plus pragmatiques, sont parfois justifiées par la crainte des collectivités de voir leur plan local d'urbanisme annulé par le juge administratif.

En dépit de la marge de manœuvre laissée aux autorités, la concertation n'en reste pas moins une procédure obligatoire prévue dans le cadre de l'adoption d'un document particulier. Dans d'autres hypothèses, les autorités sont à l'inverse seulement encouragées à développer des démarches plus participatives. C'est le cas dans le cadre de la politique de la ville. En effet, la loi de programmation pour la ville et la cohésion urbaine du 21 février 2014 ( $n^{\circ}$ 2014-173) semble faire la part belle à l'idée de participation puisque l'on retrouve à l'article $1^{\text {er }}$ le principe selon lequel la politique de la ville

[...] s'inscrit dans une démarche de coconstruction avec les habitants, les associations et les acteurs économiques, s'appuyant notamment sur la mise en place de conseils citoyens ${ }^{34}$, selon des modalités définies dans les contrats de ville, et sur la coformation ${ }^{35}$.

Le caractère pour le moins obscur et imprécis des termes employés doit être souligné ${ }^{36}$. Néanmoins, le ministère de la Ville met à disposition sur son site Internet une «boîte à outils » recensant plusieurs modalités de mise en œuvre de la participation, parmi lesquelles figurent les marches exploratoires ${ }^{37}$. Inspirées du Canada, elles

29. Art. L. 103-2 du Code de l'urbanisme.

30. J.-F. Struillou, «Participation du public aux procédures d'urbanisme», JurisClasseur Collectivités territoriales, fasc. 1022 , octobre 2015.

31. Art. L. 103-4 du Code de l'urbanisme.

32. M. Rauze, maire de Morsang-sur-Orge, «Le Conseil local d'élaboration du PLU est en place», site de la mairie de Morsang-sur-Orge, http:// morsang.fr/spip.php?article12346: «Il n’y a pas de précédent ou d'expérience de même nature auxquels nous référer. Nous allons donc travailler sans filet et beaucoup inventer».

33. M. Rauze, «CLE-PLU : méthode et calendrier», site de la mairie de Morsang-sur-Orge, http://morsang.fr/spip.php?article12503: «C’est de lui que tout part et c'est à lui que tout revient».

34. La loi de février 2014 prévoit donc la mise en place obligatoire de conseils citoyens, mais là encore, force est de constater que cette loi laisse une marge de manœuvre conséquente aux autorités; voir la loi nº 2014-173 du 21 février 2014 de programmation pour la ville et la cohésion urbaine, art. 7.

35. Loi $\mathrm{n}^{\mathrm{o}}$ 2014-173, art. $\mathrm{e}^{\mathrm{er}}$ (nous soulignons).

36. J.-L. Borloo, Assemblée nationale, séance du 22 novembre 2013; cité par J.-P. Brouant, «Ville et cohésion urbaine. La continuité dans le changement, c'est maintenant!», L'actualité juridique. Droit administratif, nº 17, mai 2014, p. 973: «Si la coconstruction, c'est du cobavardage, si elle ne s'accompagne d'aucune augmentation financière ni d'aucune politique de soutien réel, alors nous reviendrons à la situation d'il y a quinze ou vingt ans: la stratégie des grands mots. En réalité, les procédures n’auront de participatif que le nom!».

37. Site du ministère de la Ville, de la Jeunesse et des Sports, http://www.ville.gouv.fr/IMG/pdf/participation_habitants_plaquette.pdf. 
sont organisées dans le but d'améliorer la sécurité des femmes et permettent aux habitants, à l'occasion d'une marche dans un secteur particulier de la ville, d'analyser les avantages et les inconvénients éventuels rencontrés sur ce secteur. Les habitants peuvent alors faire remonter leurs constats et leurs préconisations aux autorités qui sont parfois présentes à leurs côtés lors de ces marches ${ }^{38}$. Se développent également depuis plusieurs années, selon un mode opératoire similaire mais plus spécifique à l'urbanisme et à l'aménagement, des diagnostics en marchant.

Dépourvues de cadre juridique précis mais encouragées par les pouvoirs publics, ces formes se multiplient en pratique. On pourrait également mentionner d'autres initiatives «a-juridiques», telles que les budgets participatifs, développés notamment par la mairie de Paris, qui relèvent souvent de l'aménagement, du cadre de vie et sont parfois intégrés aux conseils de quartier, obligatoires pour les communes de plus de 80 ooo habitants ${ }^{39}$. Par exemple, des Parisiens ont été à l'initiative et ont voté pour un projet de végétalisation d'un mur, la rénovation d'un square ou encore l'aménagement de toits de certains immeubles. Pour la période 2014-2020, un demi-milliard d'euros est prévu par la mairie de Paris pour financer des projets choisis dans le cadre de ces budgets participatifs ${ }^{40}$. Certains élus privilégient également le contact entre les habitants et les professionnels de l'urbanisme. En effet, se développent de plus en plus des lieux comme le Pavillon à Caen ${ }^{41}$, conçu comme un espace de rencontre, d'apprentissage, de débat ou encore de présentation de projets. Cette volonté d'échange avec les habitants mais aussi entre les habitants se retrouve également à Nantes. La ville a mis en place un «site internet du dialogue citoyen de quartier», permettant notamment aux habitants de donner leur avis et de centraliser les différents espaces de participation ${ }^{42}$. En outre, cette plateforme présente l'avantage de constituer une source d'information pour les habitants.
En pratique, ces différentes formes de participation sont essentiellement consultatives, permettant à l'habitant d'être davantage un porte-voix qu'un réel participant qui pourrait s'associer activement à la construction d'un projet, à l'élaboration d'une décision, voire à la prise de décision finale. Il existe parfois une volonté de dépasser la simple consultation pour s'inscrire dans une démarche relevant davantage de la coconstruction, mais elle laisse la plupart du temps le dernier mot aux autorités ${ }^{43}$. Or, comme nous l'avons déjà constaté, la concertation relève d'une participation essentiellement symbolique ${ }^{44}$. L'idée de participation est donc encore limitée, malgré la volonté de certains élus de renforcer son effectivité.

Ces derniers peuvent rencontrer certaines difficultés dans la mise en place des formes de participation, ce qui pourrait expliquer en partie les limites observées et le fait que certains élus soient réticents à la mettre en œuvre. C'est en effet une tâche qui peut s'avérer délicate, notamment si l'on souhaite que celle-ci soit la plus efficiente et inclusive possible ${ }^{45}$. Le rôle de ceux que l'on peut qualifier aujourd'hui de professionnels ou d'experts de la participation ${ }^{46}$ est alors crucial: sources d'idées, d'expériences à mener pour multiplier les occasions de faire participer, ils constituent également un outil de formation des élus ou encore des agents de la ville qui ne disposent pas forcément de cette "culture de travail en commun ${ }^{47}$. Les élus ont donc souvent recours «à des consultants extérieurs pour organiser et animer les dispositifs participatifs », ce qui a favorisé la naissance d' " un véritable marché de la participation ${ }^{48}$. À l'origine, les personnes chargées d'animer et de mettre en place ces dispositifs participatifs étaient plutôt des militants, des représentants du monde associatif ou encore des travailleurs sociaux. Mais progressivement, on a observé une réelle professionnalisation de cette activité, avec l'émergence d'entreprises spécialisées dans la participation, avec comme intervenants des conseillers en communication ou en management public ${ }^{49}$. Cette

38. Des «marches exploratoires» ont été organisées dans de nombreuses villes, notamment à Troyes à l’initiative de la déléguée du préfet, secrétariat général du Comité interministériel des villes; voir Guide méthodologique des marches exploratoires. Des femmes s'engagent pour la sécurité de leur quartier, Saint-Denis La Plaine, Les Éditions du CIV (Cahiers pratiques Hors-série), 2012, en ligne: http://www.ville.gouv.fr/IMG/pdf/ sgciv-guidemarcheexploratoire.pdf. Les organisateurs de ces marches sont parfois obligés de refuser des participants; voir D. Bertrand, in La gestion urbaine de proximité: une ambition et une pratique au service des habitants des quartiers (Rencontre des acteurs de la ville organisée par le secrétariat général du Comité interministériel des villes, 17 décembre 2009, Paris), Saint-Denis La Plaine, Les Éditions du CIV, 2010, en ligne: http://www.ville.gouv.fr/IMG/pdf/La_gestion_urbaine_de_proximite_actes.pdf, p. 80.

39. Art. L. 2143-1 du Code général des collectivités territoriales.

40. Voir le site de la mairie de Paris, https://budgetparticipatif.paris.fr/bp/\#.

41. http://www.lepavillon-caen.com

42. https://www.nantesco.fr/home/charte-dutilisation-1.html; le nombre de contributions sur le site de la ville est faible, mais celui-ci n’a été mis en ligne qu'en décembre 2015.

43. Une mairie peut être amenée à faire des concessions sur ses projets, par exemple en enlevant un étage sur un bâtiment (entretien avec $\mathrm{M}^{\mathrm{me}}$ Sonia de la Provôté, maire-adjointe chargée de l'urbanisme à la mairie de Caen, le 14 mars 2016).

44. Voir supra.

45. L'un des reproches courant mais néanmoins justifié adressé à la participation est l'idée qu'elle ne ferait que reproduire un schéma de type représentatif, où seule une poignée de personnes participe; voir par exemple L. Blondiaux, Le nouvel esprit de la démocratie. Actualité de la démocratie participative, Paris, Seuil, 2008, p. 63-80.

46. Voir A. Mazeaud, M. Nonjon, «Professionnel», in Dictionnaire critique et interdisciplinaire de la participation, I. Casillo, R. Barbier, L. Blondiaux, F. Chateauraynaud, J.-M. Fourniau, R. Lefebvre, C. Neveu, D. Salles (dir.), Paris, GIS Démocratie et Participation, 2013, en ligne: http://www. participation-et-democratie.fr/fr/dico/professionnel.

47. C. Touchefeu, in La gestion urbaine de proximité..., p. 68.

48. M. Nonjon, «Professionnels de la participation: savoir gérer son image militante», Politix, n 70, 2005/2, p. 90.

49. Ibid. 
professionnalisation croissante permet sans aucun doute de faciliter la mise en place de ces procédés, et ainsi, de décharger les élus de certaines contraintes. Il faut toutefois veiller à ce que cela ne conduise pas à une certaine opacification ou encore à un formatage de la participation. En effet, cela pourrait avoir pour effet de bloquer les initiatives les plus originales et d'éloigner l'autorité du dispositif et par voie de conséquence, de ses participants.

Par ailleurs, ces différentes démarches qui se développent tantôt dans le cadre des procédures réglementées de participation, tantôt en marge de ces mécanismes, sont en cours de judiciarisation, même si ce processus ne fait que commencer ${ }^{50}$. L'équilibre entre réglementation, uniformisation des pratiques et liberté des autorités est donc délicat à trouver, les pratiques étant dans le domaine variables, dépendantes du bon vouloir des élus. Se développent alors, en parallèle des éventuelles formes de participation mises en place par les élus, d'autres modes d'actions, à l'initiative des habitants cette fois.

\section{B. Les mécanismes parallèles de la participation: le développement de formes d'actions citoyennes contestataires}

Le développement d'autres formes de participation par les citoyens peut s'expliquer par les lacunes des procédés participatifs mis en place. Les processus de participation ne permettent en effet généralement pas de remettre en cause la pertinence même du projet, mais simplement de le modifier à la marge. Ils sont alors d'une utilité limitée. En outre, le sentiment de ne pas avoir été entendu, des dossiers d'enquête publique insuffisants, voire mensongers, la connivence des élus et des maîtres d'ouvrage, sont des arguments souvent invoqués par les citoyens ${ }^{51}$. Ce sentiment a été relayé par un collectif de citoyens auditionné courant 2015 par la commission sur la démocratisation du dialogue environnemental ${ }^{52}$ qui a remis un rapport contenant vingt propositions ${ }^{53}$.

Reste alors le répertoire contestataire. La palette d'actions est variée ${ }^{54}$. Souvent, elles forment un ensemble et s'inscrivent dans un cadre d'actions plus ou moins organisées. On observe alors « une nébuleuse d'actions parallèles qui se soutiennent réciproquement les unes les autres ${ }^{55}$, allant de l'action en justice, en passant par la pétition ou encore, dans certains cas, se traduisant par un appel à la désobéissance.

Les opposants à un projet d'aménagement ou d'urbanisme peuvent tout d'abord avoir recours à la justice pour se faire entendre. Certains, réunis en association ou en collectif, n'hésitent pas à utiliser le droit comme une $\operatorname{arme}^{56}$. Ce phénomène n'est pas nouveau. Dès le début des années 1990, « de nombreuses associations se sont en effet constituées à Paris comme ailleurs pour lutter [...] contre des projets immobiliers privés en cours ou projetés dans leur quartier ${ }^{57}$. Ces associations n'hésitent pas à faire des recours, notamment devant le juge administratif pour qu'il annule l'opération. D'ailleurs, les lacunes des formes réglementées de participation sont souvent invoquées pour contester la légalité de la décision, mais il semble qu'il ne soit pas toujours aisé d'obtenir satisfaction sur ce terrain ${ }^{58}$. Le recours en justice place aussi l'association ou le collectif dans une position qui peut conduire à une négociation avec les élus ${ }^{59}$. Ces acteurs ne sont en effet pas nécessairement dans une perspective de rejet de l'opération dans son ensemble, mais utilisent l'action en justice comme un levier pour être entendus. On peut à cet égard penser aux recours de l'association Tam-Tam dans le cadre de la zone d'aménagement concerté (ZAC) Paris Rive Gauche courant des années $1990^{60}$ ou plus récemment aux recours effectués contre des «grands projets inutiles ${ }^{61}$ comme la construction d'un grand centre

50. Art L. 131-1 du Code des relations entre le public et l'Administration : «Lorsque l'administration décide, en dehors des cas régis par des dispositions législatives ou réglementaires, d'associer le public à la conception d'une réforme ou à l'élaboration d'un projet ou d'un acte, elle rend publiques les modalités de cette procédure, met à disposition des personnes concernées les informations utiles, leur assure un délai raisonnable pour y participer et veille à ce que les résultats ou les suites envisagées soient, au moment approprié, rendus publics» (nous soulignons); voir également Conseil d'État, Rapport public 2011. Consulter autrement, participer effectivement, Paris, La documentation française (Études et documents du Conseil d'État; 62), 2012, p. 107.

51. Voir par exemple, pour Sivens, B. Lefetey, Sivens, un barrage contre la démocratie, Paris, Les petits matins, 2015, p. 43-56.

52. Commission spécialisée du Conseil national de la transition écologique sur la démocratisation du dialogue environnemental présidée par Alain Richard, Rapport Démocratie environnementale: débattre et décider, remis à $\mathrm{M}^{\mathrm{me}}$ Ségolène Royal, ministre de l'Écologie, du Développement durable et de l'Énergie, le 3 juin 2015.

53. Collectif citoyen, Modernisation de la «démocratie participative» - Plate-forme proposée par les mouvements d'opposition aux Grands Projets Inutile Imposés: 3 grands principes - 20 propositions concrètes, en ligne: http://www.collectif-testet.org/uploaded/D\%C3\%A9mocratie_participative/ plate-forme-finale-da-mocratie-participative.pdf.

54. On retiendra ici la manifestation ou encore la pétition, comme celle déposée sur la plateforme www.change.org contre le projet «Inter-Ikéa prévoyant l'ouverture d'un nouveau centre commercial et de loisirs à Fleury-sur-Orne, qui a rassemblé plus de 12 ooo signatures (https://www.change.org/p/ ikea-caen-fleury-sur-orne-annuler-le-projet-inter-ikea)

55. Y. Renaud, «Agir en justice. Les usages du recours juridique par les associations», Le Philosophoire, n 15, $2001 / 3, \mathrm{p} .120$.

56. Le recours au droit, à la justice, peut alors s'apparenter comme faisant partie du «répertoire de la mobilisation " (L. Israël, L'arme du droit, Paris, Presses de Sciences Po, 2009, p. 38).

57. Y. Renaud, «Agir en justice...», p. 119.

58. Voir J.-F. Struillou, «Participation du public... »; J.-F. Struillou, «La participation des habitants: la concertation de l'article L. $300-2$ du code de l'urbanisme", in Droit et politiques de renouvellement urbain, GRIDAUH (dir.), Paris, GRIDAUH (Les cahiers du GRIDAUH; 10), 2004, p. 74-79.

59. Y. Renaud, "Agir en justice...", p. 122.

60. Voir H. Nez, Urbanisme: la parole citoyenne, Lormont, Le bord de l'eau, 2015, p. 100-104.

61. Voir Camille, Le petit livre noir des grands projets inutiles, Neuvy-en-Champagne, Le passager clandestin, 2015. 
commercial à côté de Toulouse ou encore l'extension du stade Roland-Garros.

Si les citoyens tentent parfois de se faire entendre par le droit, ils ne vont parfois pas hésiter à aller contre le droit en effectuant des actions illégales revendiquées par les promoteurs de la désobéissance comme des «actions citoyennes réfléchies ${ }^{62}$. La désobéissance civile se définit selon John Rawls comme:

[...] un acte public, non violent, décidé en conscience, mais politique, contraire à la loi et accompli le plus souvent pour amener à un changement dans la loi ou bien dans la politique du gouvernement ${ }^{63}$.

En France, les désobéissants les plus notoires sont sans aucun doute les faucheurs volontaires d'OGM. Plus récemment, un acronyme est devenu tristement célèbre, notamment suite au décès d'un jeune militant écologiste à Sivens en 2014: la ZAD. Une "zone à défendre», détournement de la zone d'aménagement différé, se définit comme:

[...] une occupation illégale de parcelles de terrain, appartenant à des personnes publiques ou privées, qui a pour effet ou pour but de rendre impossible la conduite d'un projet ou d'un ouvrage implanté en tout ou partie sur lesdites parcelles ${ }^{64}$.

Forme d'appropriation du territoire, de l'espace, les «zadistes» de Notre-Dame-des-Landes s'opposent à la construction d'un aéroport ou encore à la construction d'un Center Parcs comme à Roybon dans l'Isère. Moins connue, une «ZAD patate» sur la plaine de Montesson en région parisienne a été investie par des habitants qui ont créé « un jardin pour dire non à l'urbanisation de la plaine de Montesson ${ }^{65}$.

Par ces actions, les désobéissants cherchent à alerter l'opinion et les autorités publiques, n'hésitant pas pour cela à être jugés, le procès s'apparentant d'ailleurs à une «arène militante ${ }^{66}$, un moyen de médiatiser la cause défendue.
Encore faut-il qu'ils soient entendus. On peut noter que le rapport de force créé par ce type de mouvement peut conduire les autorités à reculer. À titre d'exemple, le 24 décembre 2015, un arrêté interdépartemental signé par les préfets du Tarn et du Tarn-et-Garonne a été adopté, abandonnant le projet initial du barrage de Sivens. L'adoption de la loi du 25 juin $2008^{67}$ et celle du 2 juin $2014^{68}$ montrent que si le législateur a entendu la revendication des faucheurs, il a en revanche incriminé le mode d'action par la création d'un délit de fauchage en 2008. Ces mouvements de désobéissance peuvent donc avoir de réels effets sur la production des normes juridiques, même s'ils ne sont pas toujours ceux escomptés.

La position de l'élu local confronté à des actions de désobéissance est peu enviable comme l'explique Nicole Belloubet: Sivens constitue

[...] l'exemple type d'un piège dans lequel les décideurs doivent éviter de se laisser enfermer : l'évacuation des lieux peut souder davantage encore les manifestants; l'occupation des lieux implique soit l'abandon du projet, soit, à tout le moins, des concessions vis-à-vis de sa réalisation ${ }^{69}$.

Les actions de désobéissance expriment donc une autre forme de participation, non réglementée, plus «sauvage». Au-delà des contestations autour de certains projets particuliers, les citoyens semblent être en recherche d'une démocratie plus « réelle ${ }^{70}$ et d'un mode de vie alternatif comme on le constate dans les $\mathrm{ZAD}^{71}$.

L'élu a donc tout intérêt à mettre en place en amont des espaces permettant non seulement de consulter les habitants mais surtout de les entendre. Les mécanismes de participation se doivent, dans le domaine de l'aménagement comme dans les autres domaines, d'être plus effectifs et de dépasser les schémas traditionnels. Si les formes de participation mises en place aujourd'hui témoignent bien d'une volonté de renouveler notre fonctionnement démocratique, le chemin à parcourir est encore long ${ }^{72} \ldots$

62. J. Bové, G. Luneau, Pour la désobéissance civique, Paris, La Découverte, 2004, p. 14.

63. J. Rawls, Théorie de la justice, C. Audard (trad.), Paris, Seuil, 1987, p. 405.

64. N. Mamère, P. Popelin, Rapport fait au nom de la commission d'enquête chargée d'établir un état des lieux et de faire des propositions en matière de missions et de modalités du maintien de l'ordre républicain, dans un contexte de respect des libertés publiques et du droit de manifestation, ainsi que de protection des personnes et des biens, $\mathrm{n}^{\circ}$ 2794, enregistré à la présidence de l'Assemblée nationale le 21 mai 2015 , p. 82.

65. Site Internet du mouvement: https://zadpatate.wordpress.com/about.

66. G. Hayes, S. Ollitrault, La désobéissance civile, Paris, Presses de Sciences Po, 2013, p. 118.

67. Loi nº 2008-595 du 25 juin 2008 relative aux organismes génétiquement modifiés.

68. Loi no $2014-567$ du 2 juin 2014 relative à l'interdiction de la mise en culture des variétés de maïs génétiquement modifié.

69. N. Belloubet, «Les élus locaux confrontés à la désobéissance civile», Pouvoirs, n 155, 2015/4, p. 101.

70. Ibid., p. 102: «Ce ne sont pas seulement des revendications environnementales ou sociales qui transparaissent dans les diverses mobilisations mais des revendications liées à la démocratie».

71. Voir C. Losson, N. de La Casinière, S. Mouillard, «Dans l'écosystème zadiste», Libération, 31 octobre 2014.

72. Voir A. Ogien, S. Laugier, Le principe démocratie. Enquête sur les nouvelles formes du politique, Paris, La Découverte, 2014, p. 277: «la démocratie est toujours la démocratie à venir, le pas suivant qu'il convient de faire pour la réaliser pleinement, et cela de façon infinie». 